Original Research Paper

\title{
Modelling the Interaction between the Antenna and the Human Head by the Technique of Artificial Neural Network
}

\author{
Nizar Sghaier, Lassaad Latrach, N. Sboui, A. Gharsallah and A. Gharbi \\ Research Unit Circuits and Systems for High Frequency Electronics, Faculty of Sciences, Tunis, Tunisia
}

Article history

Received: 02-12-2014

Revised: $10-12-2014$

Accepted: 20-12-2014

Corresponding Author:

Research Unit Circuits and

Systems for High Frequency

Electronics, Faculty of Sciences,

Email: niizar.sghaier@gmail.com
Nizar Sghaier

Tunis, Tunisia

\begin{abstract}
The purpose of this study is the study and modeling of phenomenon 'interaction between the antenna and the human body' by the Artificial Neural Network (ANN). This technique is based on mathematical formulations and a base of learning who took simulations by noted trade CST MS. An example of the interaction between a body, which the dielectric properties are given and a dipole antenna, has been studied. The results validate the new approach. The good agreement between the results of the given simulation published justifies the modeling process and validates the current approach of the analysis.
\end{abstract}

Keywords: Antenna, Interaction, Human Head, ANN, CST MS, Specific Absorption Rate (SAR)

\section{Introduction}

The effect of proximity to the user's mobile phone antennas is resulting in impaired performance. The alternation is applied to the emission, adaptation, distortion. It begins with a detailed study in which we explain the interaction rather advanced between the antenna and the user. Physical alteration of matter resulting from the interaction may have biological effects (Zhadobov et al., 2011). Thus, heating of living tissue, made up mostly of water, resulting in tissue damage if the temperature rise is too intense or prolonged. Dosimetry studies, theoretical modelling and experimental measurement of ghosts are used to quantify the interaction in terms of electric and magnetic fields or local power deposited as Specific Absorption Rate (SAR).

On the other hand, we study the interaction between the antenna and the user. Several methods are encountered, namely deterministic and stochastic methods (Christodoulou and Georgiopoilos, 2001). The diversity of goals sought are given by users so that you will not find a general method of synthesis applicable to all cases, but a significant number of methods can specify the type of many problems. This diversity of solutions can be exploited to provide a useful database for a general approach to the modelling of the interaction. In this study, we present the method on neural networks that apply to the modelling approach. The neural network is used to establish the learning phase and it gives the analytical relations that are important in the step of modelling.

\section{Theory}

\section{Dielectric Properties}

Human body tissue having a depressive nature, gives many changes in dielectric properties as a function of frequency. The dielectric properties are represented by complex values and are related by the following Equation 1 (Meltz, 1995):

$$
\varepsilon^{*}(\omega)=\varepsilon^{\prime}(\omega)-j \frac{\sigma(\omega)}{\omega \varepsilon_{0}}
$$

where, denotes the real part of the permittivity which depends mainly on the frequency. The value refers to the effective value of the conductivity. It depends on the frequency and the value of the permittivity of free space. is the angular frequency which is equal to. Figure 1 shows the variation of values of and depending on the frequency.

\section{Dosimetry}

Dosimetry is to quantify the energy dissipation in an environment exposed to an electromagnetic wave, by evaluating the Specific Absorption Rate (SAR) in this environment. The SAR value is given by Equation 2 (Jokela et al., 1999):

$$
S A R=\frac{\sigma|E|^{2}}{2 \rho}
$$


With $\sigma$ and $\rho$ are referred respectively to the conductivity and the density of tissue.

The SAR is used to set exposure standards in the field of public health. Standards met are defined by the international International Commission on Non-Ionizing Radiation Protection (ICNIRP).

These effects are noticed when the power absorbed by the body exceeds the value of $4 \mathrm{~W} / \mathrm{kg}$. They result in an elevation of body temperature by 1 degree.

\section{Structure}

As the antenna is having the same behavior, that is to say, variation in its performance, which operating band involves a significant change in the interaction between the antenna and the human body within a band of operation of such an antenna. It is, then, necessary to separate the frequency dependence to evaluate the interaction. For this reason, we considered using suitable antennas as narrowband antennas wired to study the interaction (Chuang, 1994; Jensen and Rahmat-Samii, 1995). The head is modelled as a homogeneous sphere (a single tissue) of diameter $200 \mathrm{~mm}$. The dielectric properties are related to the resonant frequencies of the dipoles. Figure 2 shows the structure of the simulation (Jensen and Rahmat-Samii, 1995).

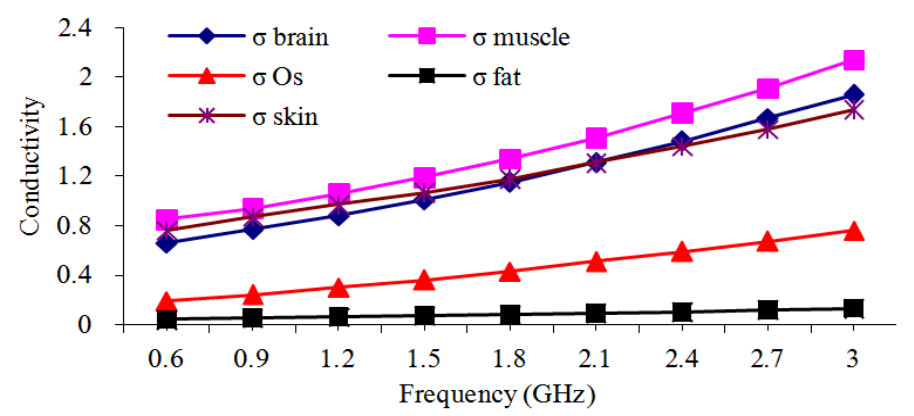

(a)

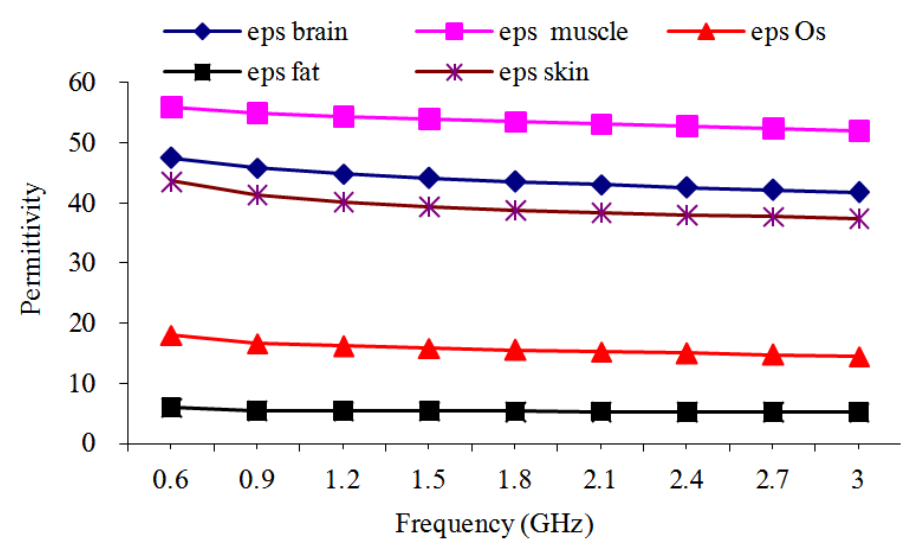

(b)

Fig. 1. All changes in dielectric properties of tissue of a human head in function of frequency (Gabriel et al., 1996) (a) Conductivity (b) Permittivity

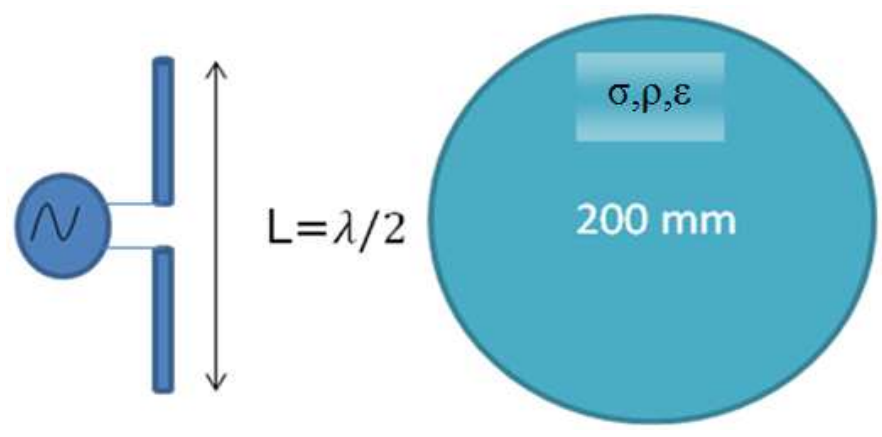

Fig. 2. Simulation structure 


\section{Neural Network Solution}

\section{Modelling}

The interaction between the antenna and the body (Chahat et al., 2012) depends on several factors. According to the working frequency, the antenna power, the power radiated by the antenna, the dielectric properties of tissues, the distance between the antenna and the head gives a look of reflection coefficient, a SAR value (between 10 and $1 \mathrm{~g}$ ), the value of radiation efficiency, the value of the input power, the input impedance. So from this explanation, we have took as input the reflection coefficient of the antenna, the working frequency, the distance $d$ between the antenna and the body, the input power, the output power, the permittivity of fabric, the fabric density and the conductivity of tissue and as an output they obtained the reflection coefficient, the SAR values averaged over 10 and $1 \mathrm{~g}$ (Hombach et al., 1996; Okoniewski and Stuchly, 1996; Kouveliotis and Panagiotou, 2006; Sager et al., 2003), the radiation efficiency, the power absorbed and the input impedance. Figure 3 shows the Neural model applied to the interaction. The Table 1 summarizes the inputs and intervals.

\section{Development of the Network Structure and Learning}

To fit our application neuronal, namely the creation of the neural network suitable for the desired application, the neural model training and testing the generalization of ANN (Chemachema and Benghalia, 2012), we use the simulation software MATLAB. Indeed, this latter provides a library rich enough to conduct this type of application known as English, Neural Network Toolbox. This library contains a various learning algorithms based on developments in their back-propagation algorithm (Acikgoz et al., 2008). Among these algorithms, there is the Trainlm. The Levenberg-Marquardt, Trainlm noted, is designed to approach an improvement in learning NAS estimated at two times faster than learning the standard algorithm based on minimizing the mean square error by descent opposite gradients of the neurons of the output layer. The learning parameters used in model training algorithm for neural Trainlm are defined as follows:

- A single hidden layer

- A number of neurons in the hidden layer which is greater than or equal to 10

- A training set which is containing 10,000 pairs of inputs and outputs

- An error threshold which is equal to the desired learning (10-6)

- A number of iterations which is equal to 1000
With one hidden layer, multilayer neural networks can generalize any data, but the problem remains to choose the number of the neurons that form the hidden layer to it, we made simulations of the number of hidden neurons that give error close to the desired error. Figure 4 shows the variation in the error based on the number of iterations by changing each time the numbers of neurons in the hidden layer.

We found that when the numbers of neurons in the hidden layer increases the error converges to a value close to the desired value. We have our goal with 16 neurons for 986 iterations.

\section{Capacity of Generalization}

In this section, we consider the S11 coefficients that are defined by a number of points. Then, we have generalized with a different number of points. Figure 5 shows the ability of generalization of a complete neural model.

Each time we have reduced the numbers of the points made in the base of learning, that with 25 simulations we can generalize 100 measurements. We are not talking on the last figure case there is an error rate under the eye.

Figure 6 shows the error rate for the previous simulations

The analysis of the error curve shows that the training set with 100 and 50 points produces an accurate generalization which means that the error is limited. These simulations are completed with a comment and as soon as the training set is wealthy, we guarantee that there are no errors. But, we are stressed, which stipulates the minimum points of the base with an acceptable error around 3\% for 25 points.

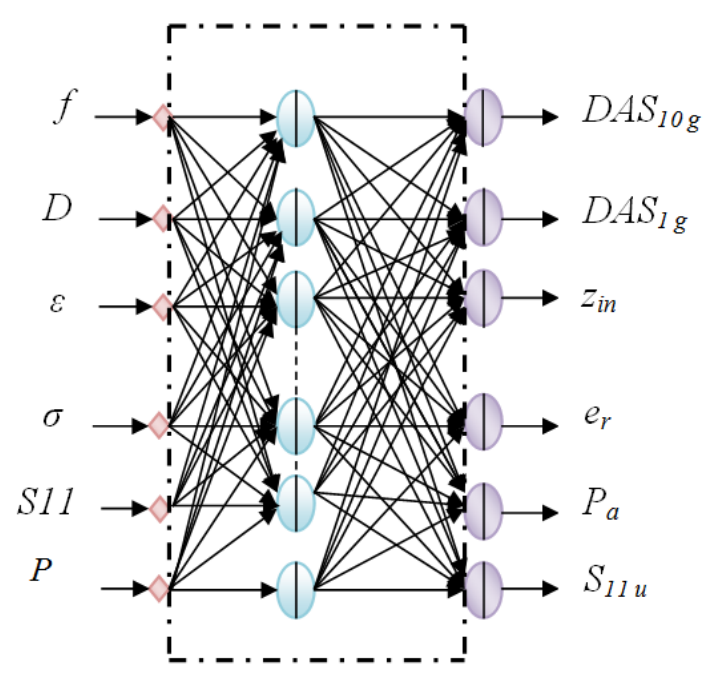

Fig. 3. Neural model applied to the interaction 
Nizar Sghaier et al. / American Journal of Engineering and Applied Sciences, 2015, 8 (3): 336.344 DOI: 10.3844/ajeassp.2015.336.344

Table 1. Inputs and intervals

\begin{tabular}{llllllr}
\hline & $\mathrm{f}(\mathrm{Ghz})$ & $\mathrm{P}(\mathrm{w})$ & $\operatorname{Pr}(\mathrm{w})$ & $\varepsilon$ & $\sigma$ & $\mathrm{S} 11$ \\
$\mathrm{~m}$ & 0.6 & 0.1 & 0.1 & Tissues & Tissues & Antennas \\
$\mathrm{M}$ & 3.0 & 5.0 & 2.0 & & & 60 \\
\hline
\end{tabular}

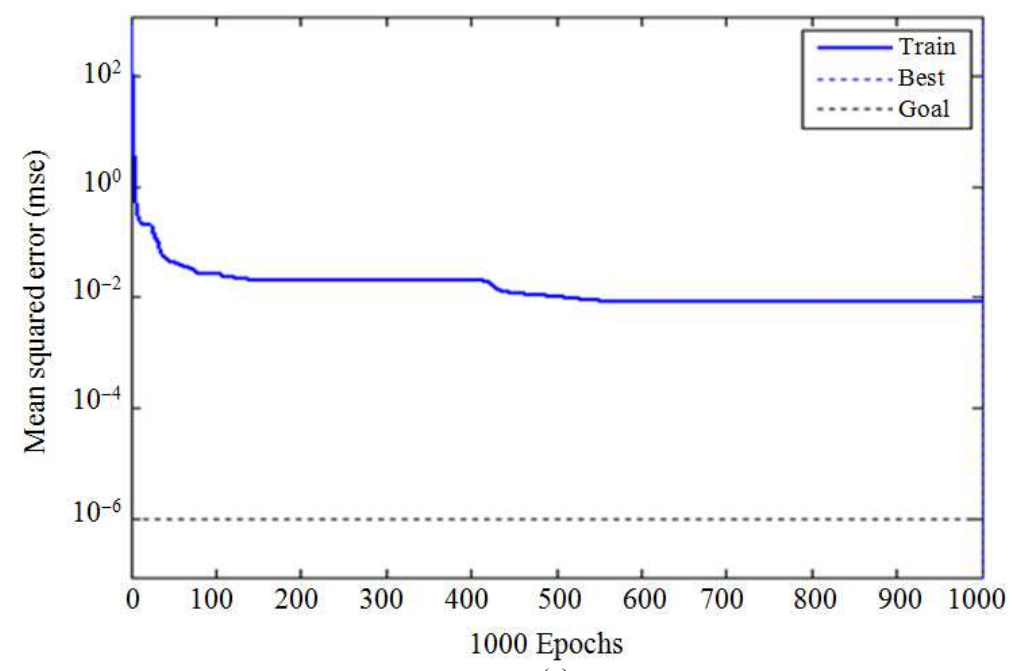

(a)

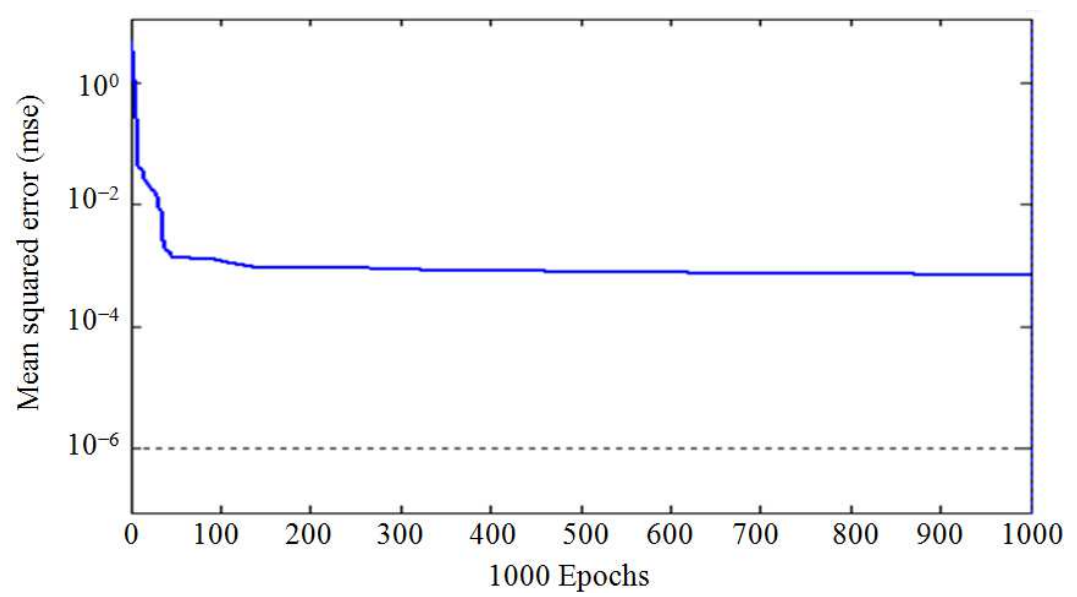

(b)

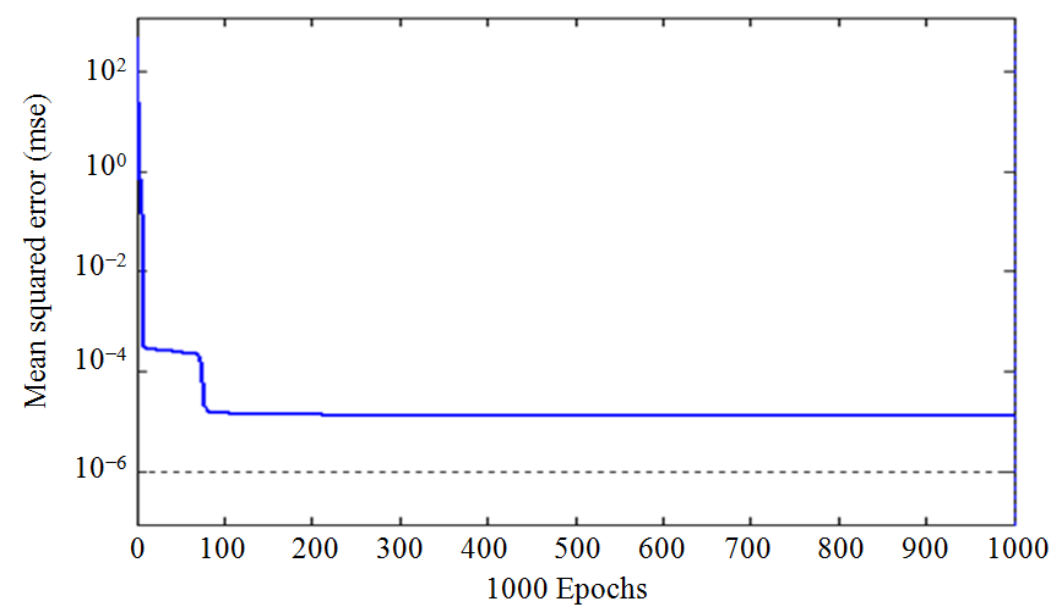

(c) 


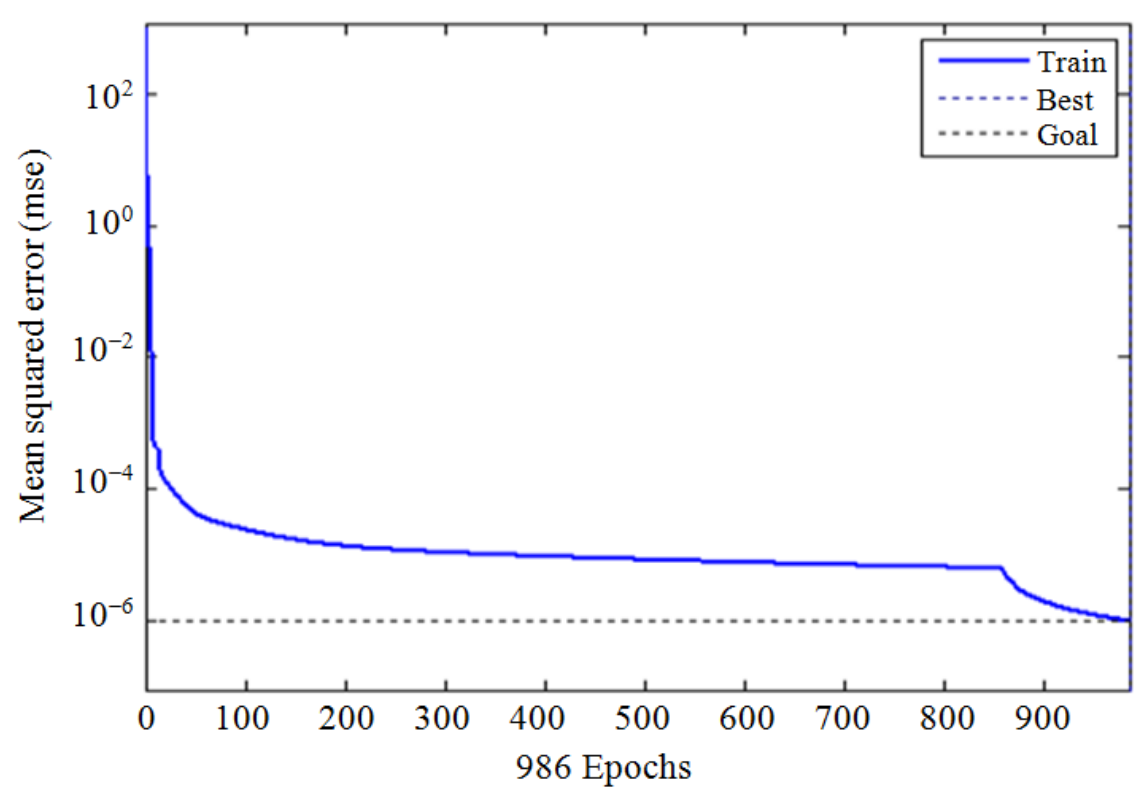

(d)

Fig. 4. Learning the neural model (a) 10 hidden neurons (b) 12 hidden neurons (c) 14 hidden neurons (d) 16 hidden neurons
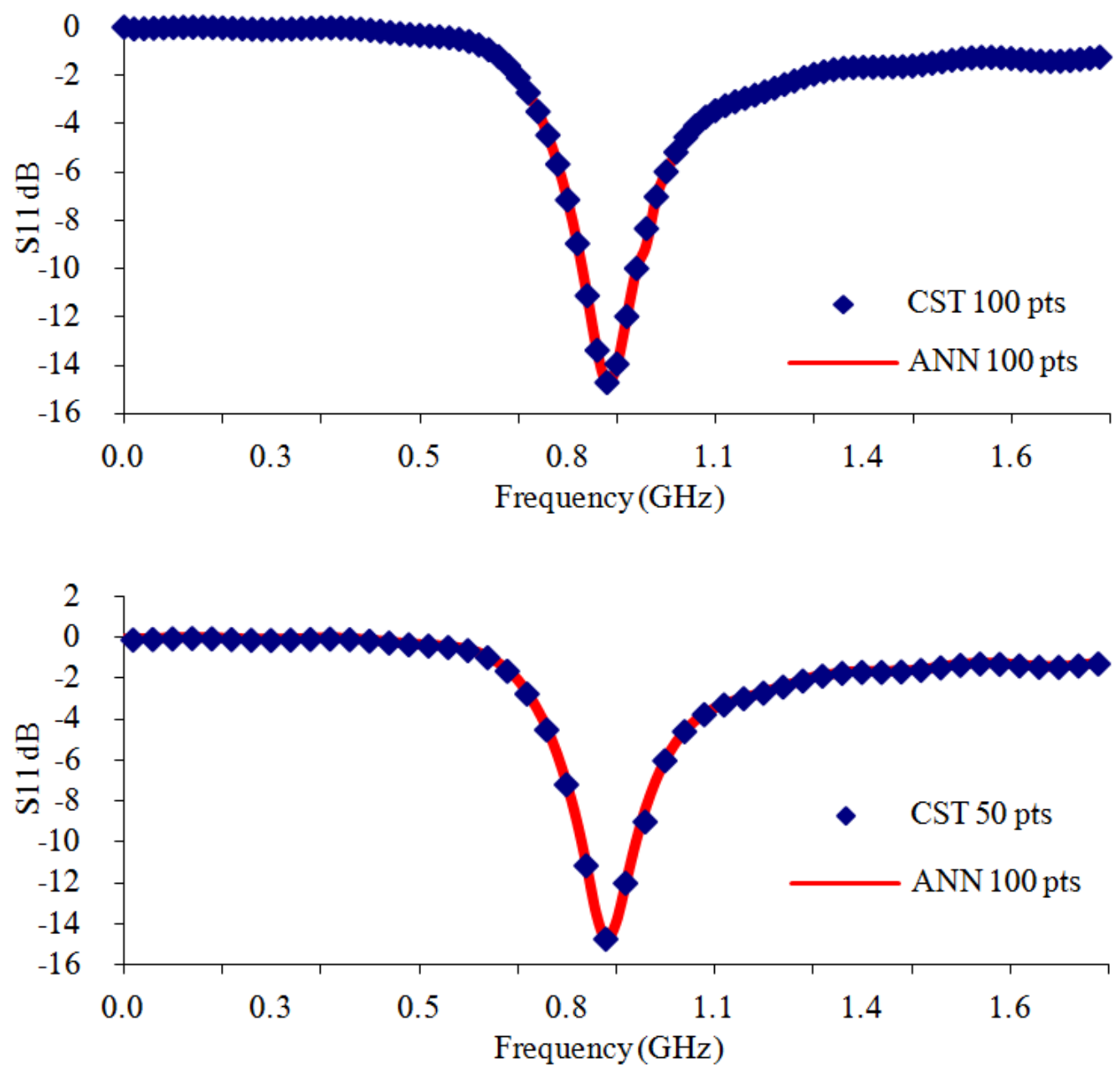

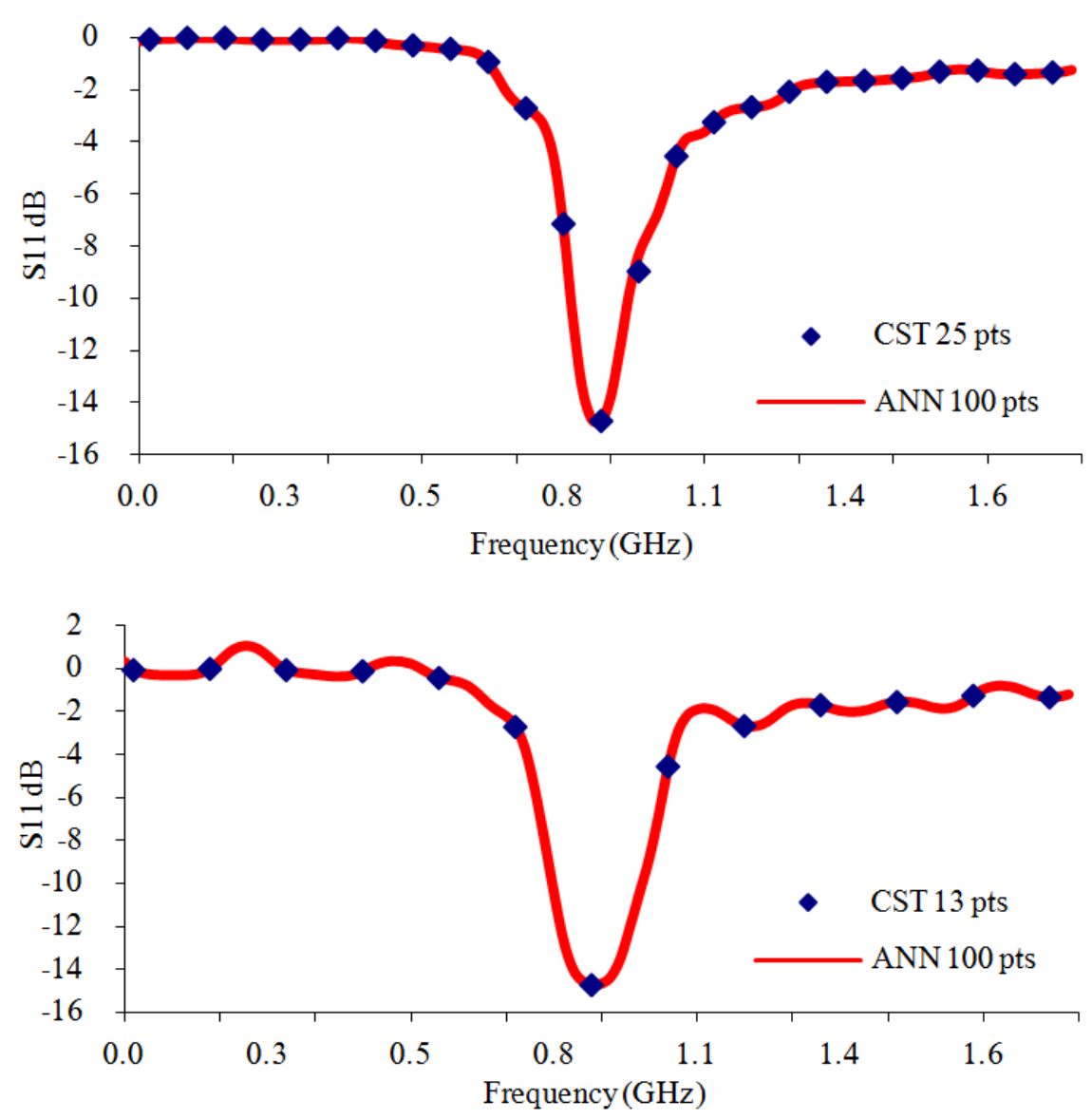

Fig. 5. Reflection coefficient for different number of points

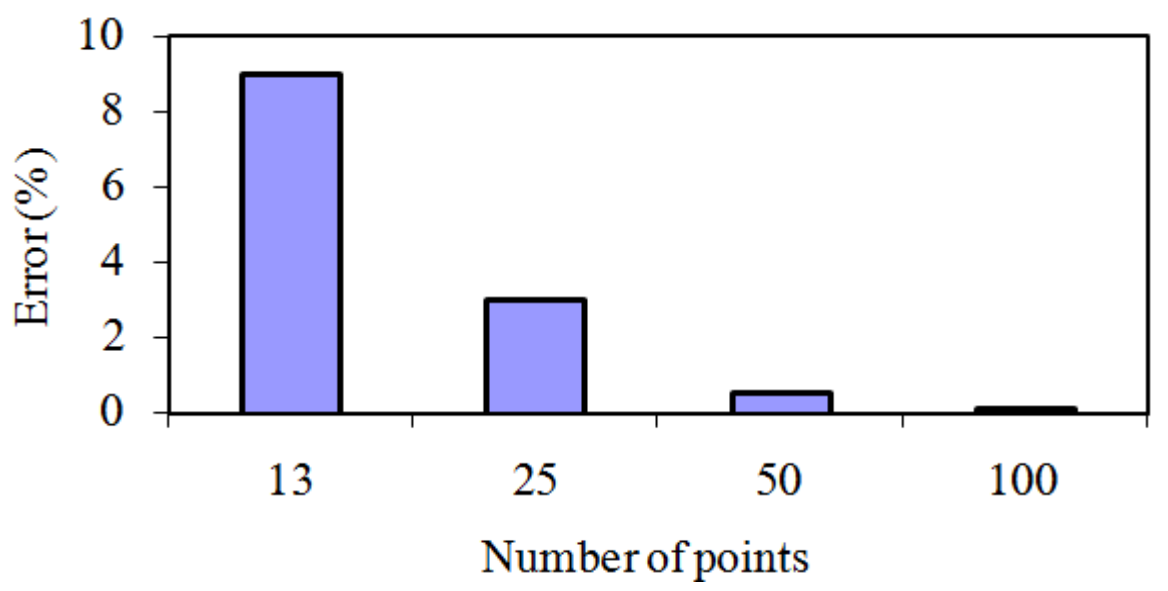

Fig. 6. Average error

\section{Results}

After neuronal validation of our model, we modeled the interaction between human bodies including the dielectric properties: $\varepsilon=42.29, \sigma=0,996$ and an antenna dipole whose characteristics $\mathrm{f}=0,905 \mathrm{GHz}, \mathrm{P}=1 \mathrm{~W}$ and $\operatorname{Pr}=350 \mathrm{~mW}$.
The neuronal model dedicated to the modeling of the interaction has a learning database of 15 points with the multi-input and the multi-output satisfy the need (75 points generalization). The simulation results illustrated in Fig. 7 confirm a good agreement with that obtained by CST MS. 

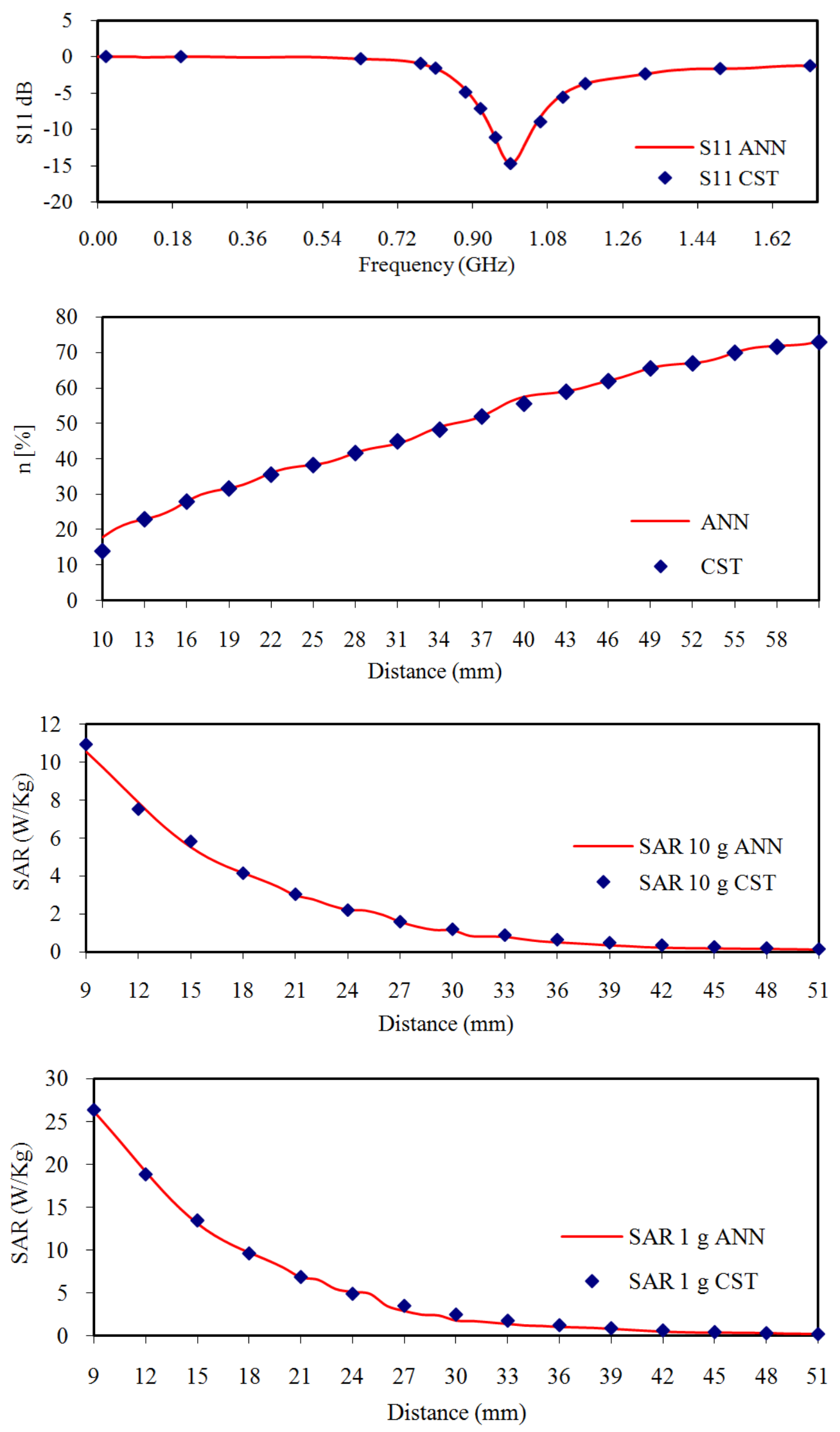

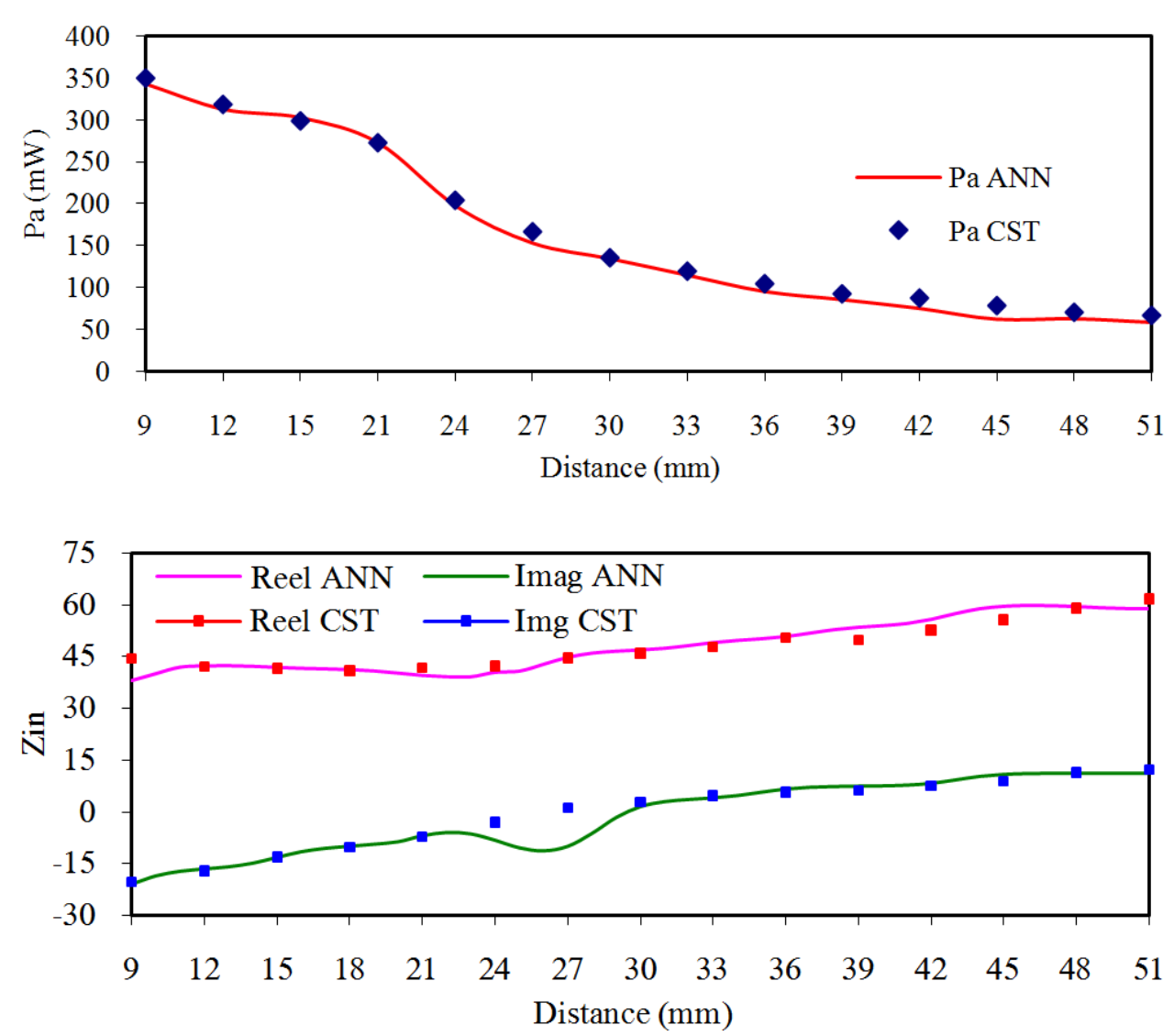

Fig. 7. Simulation results on generalized $75 \mathrm{pts}$

Table 2. Performances

\begin{tabular}{lll}
\hline Criterion type & CST MS & ANN \\
\hline Time & 5 min and $3 \mathrm{~s}$ & $13 \mathrm{~s}$ \\
2 GB memory & $60 \%$ & $17.8 \%$ \\
\hline
\end{tabular}

From the Table 2, we notice that we have a gain in terms of time with low consumption of computer memory used.

\section{Conclusion}

In this study, we have developed the modeling technique of the interaction from only weights. The multilayer neural network feed forward type is used, in particular, the perceptron multilayer MLP (Linden and Kindermann, 1989), as this type is suitable in our working. In this study, we explain the modeling of interaction using the neural network. The neural approach reduces to the extreme time calculation during the phase of use or the generalization. The model precision depends on the database of learning. However, the multilayer neural networks have some the drawbacks which are the non-speed due to the phase learning and the absence of a rule to define the network architecture. Also the major problem of the methods digital convergence, we thought to use the iterative method for the study of physical phenomena such as interaction.

\section{Acknowledgment}

I would like to express my deepest thanks to Dr. Ali Gharsallah, my thesis Director, to have me box and testified of his confidence in this study.

\section{Author's Contributions}

Nizar Sghaier: At first he designed, simulate circuits studied with commerce software CST MWS then validated the results found with the neural network approach and coordinated the data-analysis and contributed to the writing of the manuscript.

Lassaad Latrach: Developed the area of research and organized the study and contributed to the writing of the manuscript.

N. Sboui: Designed the research plan and organized the study.

A. Gharsallah and A. Gharbi: Coordinated the mouse work. 


\section{Ethics}

This article is original and contains unpublished material. The corresponding author confirms that all of the other authors have read and approved the manuscript and no ethical issues involved.

\section{References}

Acikgoz, H., Y. Le Bihan, O. Meyer and L. Pichon, 2008. Microwave characterization of dielectric materials using bayesian neural networks. Progress Electromagne. Res., C3: 169-182. DOI: 10.2528/PIERC08030603

Chahat, N., M. Zhadobov, L. Le Coq, S.I. Alekseev and R. Sauleau, 2012. Characterization of the interactions between a $60-\mathrm{GHz}$ antenna and the human body in an off-body scenario. IEEE Trans. Antennas Propag., 60: 5958-5965. DOI: 10.1109 /TAP.2012.2211326

Chemachema, K. and A. Benghalia, 2012. Neural models for input impedance of aperture coupled rectangular patch antenna. Int. Rev. Aerospace Eng., 5: 89-89.

Christodoulou, C. and M. Georgiopoilos, 2001. Applications of Neural Networks in Electromagnetics. 1st Edn., Artech House, ISBN-10: 0890068801, pp: 512.

Chuang, H.R., 1994. Human operator coupling effects on radiation characteristics of a portable communication dipole antenna. IEEE Trans. Antennas Propagat., 42: 556-560. DOI: $10.1109 / 8.286229$

Gabriel, S., R.W. Lau and C. Gabriel, 1996. The dielectric properties of biological tissues: III. Parametric models for the dielectric spectrum of tissues. Phys. Med. Biol., 41: 2271-2293. DOI: $10.1088 / 0031-9155 / 41 / 11 / 003$

Hombach, V., K. Meier, M. Burkhardt, E. Kühn and N. Küster, 1996. The dependence of EM energy absorption upon human head modeling at 900 MHz. IEEE Trans. Microwave Theory Techn., 44: 1865-1873. DOI: $10.1109 / 22.539945$
Jensen, M.A. and Y. Rahmat-Samii, 1995. EM interaction of handset antennas and a human in personal communications. Proc. IEEE, 83: 7-17. DOI: $10.1109 / 5.362755$

Jokela, K., D. Leszczynski, W. Paile, S. Salomaa and L. Puranen, 1999. Radiation safety of mobile phones and base stations. Radiation and Nuclear Safety Authority, Helsinki.

Kouveliotis, N.K. and S.C. Panagiotou, 2006. Theoretical approach of the interaction between a human head model and a mobile handset helical antenna using numerical methods. Progress Electromagnet. Res., 65: 309-327. DOI: $10.2528 /$ PIER06101901

Linden, A. and J. Kindermann, 1989. Inversion of multilayer nets. Proceedings of the International Joint Conference on Neural Networks, Jun. 18-22, IEEE Xplore Press, Washington D.C, pp: 11425-11430. DOI: 10.1109/IJCNN.1989.118277

Meltz, M.L., 1995. Biological Effects Versus Health Effects: An Investigation of the Genotoxicity of Microwave Radiation. In: Radiofrequency Radiation Standards: Biological Effects, Dosimetry, Epidemiology and Public Health Policy, Klauenberg, B.J., M. Grandolfo and D.N. Erwin (Eds.), Springer, ISBN-10: 0306449196, pp: 235-241.

Okoniewski, M. and M.A. Stuchly, 1996. A study of the handset antenna and human body interaction. IEEE Trans. Microwave Theory Techn., 44: 1855-1864. DOI: $10.1109 / 22.539944$

Sager, M., M. Forcucci and T. Kristensen, 2003. A novel technique to increase the realized efficiency of a mobile phone antenna placed beside a headphantom. Proceedings of the IEEE Antennas and Propagation Symposium, Jun. 22-27, IEEE Xplore Press, Columbus, OH, USA., pages 1013-1016. DOI: 10.1109/APS.2003.1219406

Zhadobov, M., N. Chahat, R. Sauleau, C. Le Quement and Y. Le Drean, 2011. Millimeter-wave interactions with the human body: State of knowledge and recent advances. Int. J. Microwave Wireless Technol., 3: 237-247. DOI: $10.1017 / \mathrm{S} 1759078711000122$ 